\title{
Thermal Steady States of Gases in a Gravitational Field
}

\author{
Kunihiko Kigoshi \\ Shirakawa Analysis Center, Institute of Accelerator Analysis Ltd., \\ 6-270 Ichiridan Shirasaka Shirakawa City, Fukushima 961-0835, Japan
}

Reprint requests to K. K.; Fax: +81-248-21-1057; E-mail: kigoshi@rk2.so-net.ne.jp

Z. Naturforsch. 66a, 123 - 133 (2011); received July 6, 2009 / revised January 25, 2010

\begin{abstract}
This paper presents results on observations of a temperature difference between the top and bottom of a vessel filled with gas in a gravitational field. The observed temperature at the top of the vessel was always lower than the temperature at the bottom of the vessel, and this temperature difference was persistent and steady over more than $20 \mathrm{~h}$. The magnitude of the temperature difference depends on the types of gas molecules present but is independent of the gas pressure in the vessel within the range from $2.7 \times 10^{4} \mathrm{~Pa}$ to $27 \mathrm{~Pa}$. A temperature difference between the top and the bottom is only observed along the vertical direction and is only observed when the vessel contains a gas. These experimental results indicate a gravity effect on molecular heat transfer which enables the transport of energy in the gas without a thermal gradient.
\end{abstract}

Key words: Gas in Gravitational Field; Effect of Gravity on Gas.

\section{Introduction}

The thermal steady states of gases in a gravitational field are usually considered to have uniform temperature along the direction of the gravitational force. If a gas contains different types of gas molecules, it is generally considered that fractionation of the molecules occurs along the direction of the gravitational force. For gravitational effects on the steady state of a gaseous system, the author could not find any previous reports on experimental investigations to determine the existence of a vertical thermal gradient in a gaseous system. Investigations on gravitational separation of molecules in a gas mixture were initiated two centuries ago by I. L. Gay-Lussac. However, reports presenting a proof of such separation appeared only about 20 years ago. These include analysis of gases trapped in polar ice [1], analysis of gases trapped in Antarctic and Greenland ice [2], gas preserved in Greenland ice [3], air in the lower atmosphere at Borrego Sink 1 [4], and major atmospheric components in the stratosphere [5].

Based on general understanding of the steady state of a gas in a gravitational field, a gaseous system falls to a stable state with minimum free energy [6]. However, the gas in a gravitational field has a vertical pressure gradient which makes the free energy of the system ambiguous. Sommerfeld [7] calculated the thermal state of a gas system with a vertical pressure gradient arguing that the steady state of an isolated gaseous system corresponds to its entropy maximum state. The entropy of a gaseous system with a given total energy including potential energy was calculated under the assumption that the movement of the center of gravity of the gas in the container alters the potential energy of the system. This means that the container of such a gaseous system is fixed in the coordinates of the gravitational field. Moving the center of gravity of the gas in the container changes the potential energy of the system and also changes the gravitational field outside the system. If we can neglect this change in the gravitational field, the system becomes an isolated system and its entropy maximum state corresponds to its steady state. Since the entropy maximum state of this gaseous system has no vertical thermal gradient, Sommerfeld concluded that the gas in a gravitational field also has no vertical thermal gradient.

If instead of the container, the center of gravity of the gaseous system was fixed in the coordinate system of the gravitational field, the movement of the center of gravity of the gas gives no change in the gravitational field outside the gaseous system. In this case the state of entropy maximum has a thermal gradient along the gravitational force, as will be shown in the last section of this report. The expected vertical thermal gradient of this isolated system is the same as it appears in the convective equilibrium state [8], and the top of the system is always colder than the bottom. Thus, statistical 
thermodynamics seems to give ambiguous predictions of the thermal equilibrium state for an isolated gaseous system in a gravitational field.

\section{Experimental}

In order to obtain information on the thermal steady state of a gas in a gravitational field, the temperature difference between the top and the bottom of the vessel filled with gas was measured. The vessel was kept in a high vacuum and in a strictly controlled homogenous thermal environment. If the steady state of the gas has a homogeneous temperature, the gas must have the same temperature for the controlled ambient temperature. Since the gas in the vessel has no thermal effect on the wall of the vessel, the temperature of the wall of the flask must have the same temperature as that of the flask without the gas. If we could experimentally prove that the top and the bottom temperatures of the vessel are the same, the steady state of the gas in a gravitational field has no thermal gradient along the gravitational force. If, instead, a temperature difference between the top and the bottom of the vessel is detected which is obtained only when the gas is in the vessel, then the isolated gas in the vessel has a nonhomogeneous thermal steady state.

The experiment requires a uniform and well controlled thermal environment of the vessel and precise measurement of the temperature difference between the top and the bottom. In this study, two thermistors A and $B$ were fixed on the inner surface of the flask at opposite sides. The flask can be rotated by $180^{\circ}$ around a horizontal axis located at the center of the flask. This $180^{\circ}$ rotation of the flask exchanges the positions of the two thermistors A and B in the flask. After the gas reached its thermal steady state, the temperatures at the top and the bottom of the vessel were simultaneously measured by $\mathrm{A}$ and $\mathrm{B}$ as $T_{\mathrm{A}}$ and $T_{\mathrm{B}}$, respectively. The temperature difference is denoted as $\Delta T$ $\left(=T_{\mathrm{A}}-T_{\mathrm{B}}\right)$. In order to reduce the measurement errors of $T_{\mathrm{A}}$ and $T_{\mathrm{B}}$, we used half the difference between two values of $\Delta T$ measured by $\mathrm{A}$ and $\mathrm{B}$, respectively, before and after the $180^{\circ}$ turn of the vessel. The obtained temperature difference includes errors from the measurements of the temperature difference by only one thermometer.

\subsection{Apparatus}

In the experiment, the vessel of the gas was a 4 liter crown glass spherical flask set in an aluminum drum,

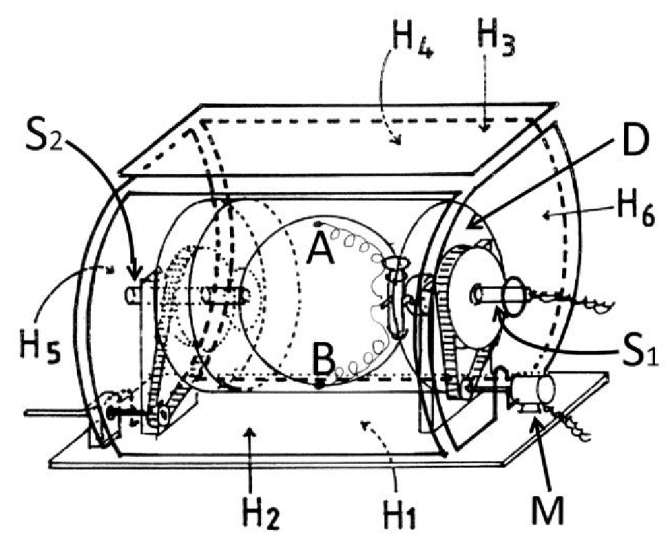

Fig. 1. Schematic of the measurement system used. A spherical crown glass flask with a cock having two thermistors A and $\mathrm{B}$, fixed respectively at the inside opposite sides of the diameter of the flask. At the center of the flask, shafts $S_{1}$ and $\mathrm{S}_{2}$ enable rotating the flask. The motor $\mathrm{M}$ drives this turning which changes the relative positions of the two thermistors A and $\mathrm{B}$. If $\mathrm{A}$ and $\mathrm{B}$ occupy positions at the top and the bottom of the flask, respectively, after a $180^{\circ}$ turn of the flask, $\mathrm{A}$ and $\mathrm{B}$ take their position at the bottom and the top of the flask, respectively. The flask was covered by the aluminum drum D. Drum D was surrounded by six heating panels $\mathrm{H}_{1}$, $\mathrm{H}_{2}, \mathrm{H}_{3}, \mathrm{H}_{4}, \mathrm{H}_{5}$, and $\mathrm{H}_{6}$. Each panel had its own thermometer and its temperature is controlled by a computer.

as shown in Figure 1. The temperature difference between the top and the bottom of the vessel was measured by the two thermistors A and B which were fixed on the inner surface of the flask. The distance between the two thermistors A and B was $19 \mathrm{~cm}$. The electric resistance of the thermistors used in this experiment is about $3 \mathrm{k} \Omega$ at room temperature. The difference in the electric resistance of $\mathrm{A}$ and $\mathrm{B}$ was measured by a 0.6 or $1.2 \mathrm{~V}, 4 \times 10^{-4} \mathrm{sec}$ square pulse. The mean power consumption of each thermistor in the flask was about $(2$ or 8$) \times 10^{-10} \mathrm{~W}$. The detection limit of the temperature difference between the thermistors $\mathrm{A}$ and $\mathrm{B}$ was $1 \times 10^{-5}{ }^{\circ} \mathrm{C}$ for a power consumption of $8 \times 10^{-10} \mathrm{~W}$ and $4 \times 10^{-5}{ }^{\circ} \mathrm{C}$ for a power consumption of $2 \times 10^{-10} \mathrm{~W}$. We confirmed that the difference in the power consumption at the thermistors within the above range had no effect on the measurement results.

The thermal environment of the flask was controlled by the temperature of the inner surface of the aluminum drum which covers the flask. The aluminum drum was boxed in six heating panels $\mathrm{H}_{1}, \mathrm{H}_{2}, \mathrm{H}_{3}, \mathrm{H}_{4}$, $\mathrm{H}_{5}$, and $\mathrm{H}_{6}$, arranged as shown in Figure 1. The temperatures of these heating panels control the heat flow from the aluminum drum to the outside environment. 


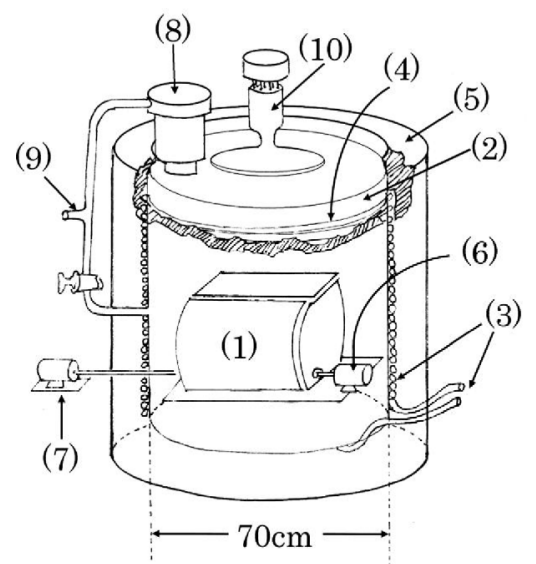

Fig. 2. Schematic of the arrangement of the apparatus. The measurement system (1) shown in Figure 1 was in a vessel of $70 \mathrm{~cm}$ height, $70 \mathrm{~cm}$ diameter iron cylinder (2). The temperature of the wall of the cylinder was kept at $24.0 \pm 0.1{ }^{\circ} \mathrm{C}$ by the circulation of constant temperature water through a vinyl tube (3) which was coiled outside the iron vessel (4). The outside of a vinyl tube was thermally insulated by Styrofoam (5). The motor (6) drives the turning of the flask and (7) drives the rotation of the aluminum drum. The iron vessel was evacuated by a turbo molecular pump (8) and a rotary pump connected to the vacuum line (9). A vacuum gauge (10) was set on the top of the glass lid.

Each heating panel has its own thermometer and the temperature is controlled by a computer to maintain a uniform and constant temperature of the aluminum drum. The temperature of the inner surface of the aluminum drum was equalized by continuous rotation of the drum.

This measurement system was placed in a cylindrical iron vessel which was evacuated by a turbomolecular pump as shown in Figure 2. The temperature of the wall of the iron vessel was kept at $24.0 \pm$ $0.1^{\circ} \mathrm{C}$ by the circulation of constant temperature water through a vinyl tube coiled around the outside of the iron vessel.

\subsection{Aluminum Drum as a Tool to Improve Thermal Environment of the Flask}

In order to measure the reduction in thermal nonuniformity in the environment of the flask by the aluminum drum, we created a horizontal thermal gradient in the outside environment of the drum by setting the temperatures of heating panels $\mathrm{H}_{1}, \mathrm{H}_{3}, \mathrm{H}_{5}$, and $\mathrm{H}_{6}$ to $24.30 \pm 0.01{ }^{\circ} \mathrm{C}, \mathrm{H}_{2}$ to $24.55 \pm 0.01{ }^{\circ} \mathrm{C}$, and $\mathrm{H}_{4}$ to $24.05 \pm 0.01{ }^{\circ} \mathrm{C}$. With this horizontal $0.5{ }^{\circ} \mathrm{C}$ temperature difference in the outside environment of the drum, the thermistors A and B set on a horizontal line in the flask gave a temperature difference of $2.8 \times 10^{-3}{ }^{\circ} \mathrm{C}$. When the drum was rotating with an angular velocity $\omega$ of $0.14 \mathrm{rad} / \mathrm{sec}$, the same $0.5{ }^{\circ} \mathrm{C}$ temperature difference was measured as $2 \times 10^{-4}{ }^{\circ} \mathrm{C}$ by thermistors $\mathrm{A}$ and $\mathrm{B}$ which were on a vertical line. However, when the thermistors were on a horizontal line, no temperature difference was detected indicating that the angular pattern of the non-uniformity of the thermal environment of the flask was rotated $\pi / 2$ from the original pattern by the continuous rotation of the drum.

In order to obtain details of the reduction in thermal non-uniformity for the temperature of the wall of the cylinder (see Fig. 3) rotating in vacuum with angular velocity $\omega$, the following analysis was carried out. We assume the thermal environment outside the cylinder to be

$$
T_{\mathrm{exD}}=T_{0}+\Delta T_{\mathrm{exD}} \cos \vartheta
$$

where $T_{\text {exD }}$ is the temperature of the outside of the cylinder, $T_{0}$ is the mean temperature of the outside environment of the cylinder, $\Delta T_{\mathrm{exD}}$ is half the maximum temperature difference in the outside environment of the cylinder, and $\vartheta$ is the angular coordinate as shown in Figure 3. The calculations of the temperature at the wall of the cylinder $\left(T_{\mathrm{D}}\right)$ under thermal steady state are

$$
\begin{aligned}
& T_{\mathrm{D}}=T_{0}+\alpha_{\mathrm{D}} \Delta T_{\mathrm{exD}} \cos (\vartheta-\psi), \\
& \alpha_{\mathrm{D}}=4 \sigma \varphi_{\mathrm{ex}} T_{0}^{3} / A^{1 / 2}, \\
& \psi=\sin ^{-1}\left(H_{\mathrm{D}} \omega / A^{1 / 2}\right), \\
& A=\left(H_{\mathrm{D}} \omega\right)^{2}+\left(\lambda_{\mathrm{D}} / R_{\mathrm{D}}^{2}+4 \sigma\left(\varphi_{\mathrm{ex}}+\varphi_{\mathrm{i}}\right) T_{0}^{3}\right)^{2},
\end{aligned}
$$

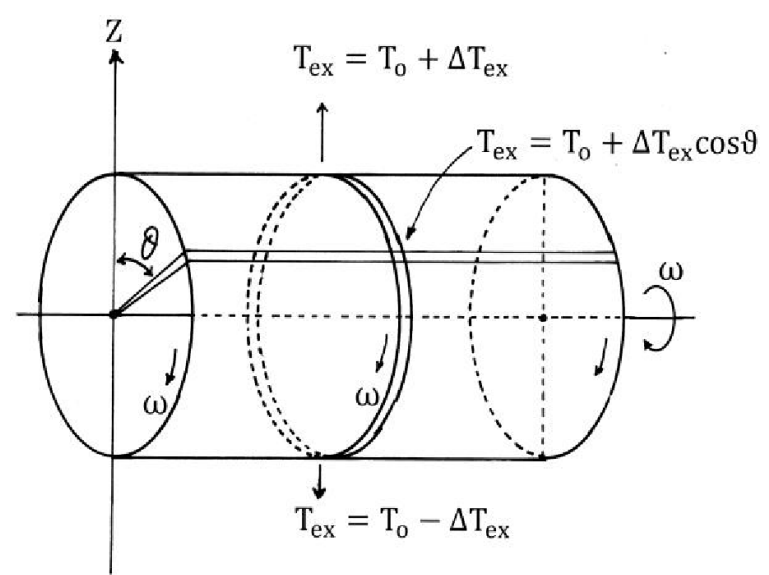

Fig. 3. Coordinate system of a cylinder which was used as a simplified model of the aluminum drum. 


\begin{tabular}{llr}
\hline Symbol Stand for & Magnitude \\
\hline$H_{\mathrm{D}}$ & Heat capacity of unit area of wall of the drum & $0.36 \mathrm{~J} / \mathrm{cm}^{2 *}$ \\
$H_{\mathrm{w}}$ & Heat capacity of unit area of wall of the flask & $0.28 \mathrm{~J} / \mathrm{cm}^{2 *}$ \\
$R_{\mathrm{D}}$ & Radius of the drum & $13 \mathrm{~cm}$ \\
$R_{\mathrm{f}}$ & Radius of the flask & $9.5 \mathrm{~cm}$ \\
$\lambda_{\mathrm{D}}$ & Thermal conductivity of wall of the drum along surface & $0.36 \mathrm{~W} / \mathrm{K}^{*}$ \\
$\lambda_{\mathrm{s}}$ & Thermal conductivity of wall of the flask along surface & $0.0011 \mathrm{~W} / \mathrm{K}^{*}$ \\
$\omega$ & Angular velocity of rotation of the drum & $0.14 \mathrm{rad} / \mathrm{sec}$ \\
$\tau$ & Thermal relaxation time of the flask & $410 \pm 10 \mathrm{sec}$ \\
$\varphi$ & Absorption and emission coefficient of thermal radiation & \\
$\varphi_{\mathrm{ex}}$ & $\varphi$ of outside surface of the drum & 0.04 \\
$\varphi_{\mathrm{i}}$ & $\varphi$ of inside surface of the drum & 0.85 \\
$\varphi_{\mathrm{f}}$ & $\varphi$ of outside surface of the flask & $0.9 *$ \\
\hline
\end{tabular}

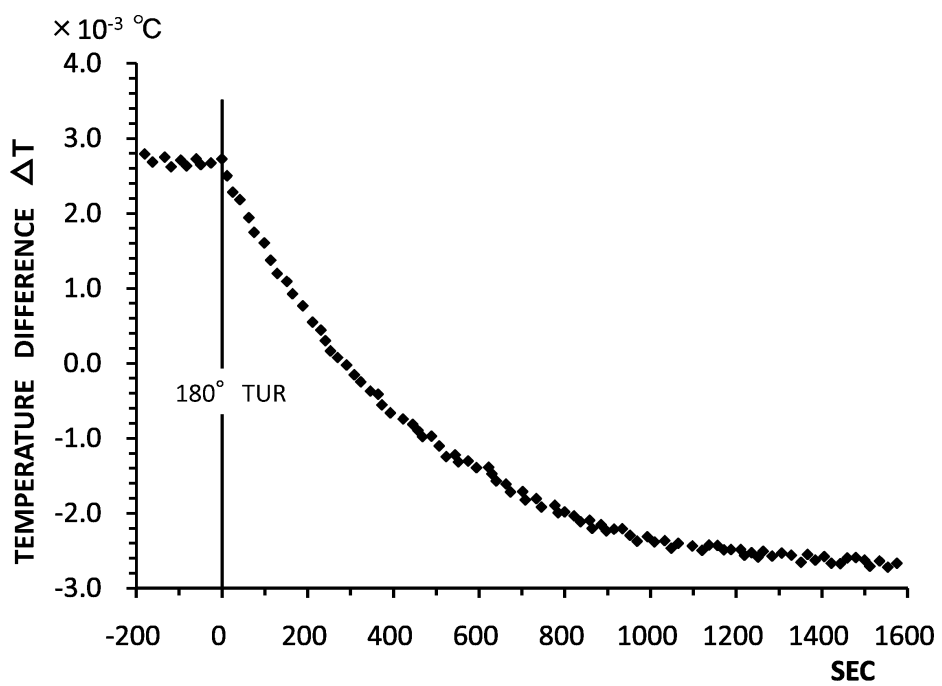

Table 1. Symbols used and their magnitudes.

* Estimated value.
Fig. 4. Thermal history of the temperature difference $\Delta T$ measured by $\mathrm{A}$ and $\mathrm{B}$ in the flask. Two thermistors $\mathrm{A}$ and $\mathrm{B}$ were set on a horizontal line in the flask. The flask was first thermally equilibrated with the thermal environment with a $0.5{ }^{\circ} \mathrm{C}$ temperature difference between the heating panels $\mathrm{H}_{2}$ and $\mathrm{H}_{4}$. At time $t=0$, the flask was turned $180^{\circ}$. The thermal history after this turn is shown by this figure plotting the change of $\Delta T$ (temperature measured by $\mathrm{A}$ - temperature measured by B). where $H_{\mathrm{D}}, \lambda_{\mathrm{D}}, R_{\mathrm{D}}, \varphi_{\mathrm{ex}}, \varphi_{\mathrm{i}}$ are listed in Table $1, \sigma$ is the Stefan-Boltzmann constant, and $\psi$ is the shift angle of the angular pattern of the thermal non-uniformity in $T_{\mathrm{D}}$. Using the numerical values listed in Table 1 , (3) and (5) become

$$
\begin{aligned}
& \alpha_{\mathrm{D}}=2.39 \times 10^{-5} / A^{1 / 2} \\
& A=(0.35 \omega)^{2}+\left(2.66 \times 10^{-3}\right)^{2} \mathrm{~W} / \mathrm{Kcm}^{2} .
\end{aligned}
$$

Without rotation $(\omega=0)$, the reduction factor is $\alpha_{D}=$ $1 / 111$. However when the drum is rotating with an angular velocity of $\omega=0.14 \mathrm{rad} / \mathrm{sec}$, then $\alpha_{\mathrm{D}}=5 \times 10^{-4}$ and shift angle $\psi=\pi / 2$.

\subsection{Thermal Characteristics of the Flask}

Figure 4 shows the change in the temperature difference measured by thermistors A and B in the flask set on a horizontal line. At first, the flask was kept for a long time in a thermal environment with temperatures of the heating panels $\mathrm{H}_{1}, \mathrm{H}_{3}, \mathrm{H}_{5}$, and $\mathrm{H}_{6}$ at $24.30 \pm 0.01{ }^{\circ} \mathrm{C}, \mathrm{H}_{2}$ at $24.55 \pm 0.01{ }^{\circ} \mathrm{C}$, and $\mathrm{H}_{4}$ at $24.05 \pm 0.01^{\circ} \mathrm{C}$. The drum boxed by these heating panels was not rotated. Inside the drum, there was a thermal gradient along the horizontal direction. After the thermal state of the flask equilibrated with that of the drum, the flask was rotated $180^{\circ}$. Subsequent to this rotation, the temperature at the wall of the flask changed to a new thermal equilibrium state. This change of temperature is plotted in Figure 4. The observed temperature change could be fitted well to an exponential decay curve and gave a thermal relaxation time of the flask of $410 \pm 10 \mathrm{sec}$.

At first, the flask was kept for a long time in the thermal environment of $T_{\text {exfo }}$ given by

$$
T_{\text {exf } 0}=T_{0}+\Delta T_{\text {exf }} \cos \vartheta,
$$

where $\Delta T_{\mathrm{exf}}$ is half the horizontal temperature differ- 


\begin{tabular}{|c|c|c|c|c|c|}
\hline $\begin{array}{l}\text { Sample } \\
\text { gas }\end{array}$ & $\begin{array}{l}\text { Press } \\
/ \mathrm{Pa}\end{array}$ & $\begin{array}{l}{[\Delta T]_{\text {turn }}} \\
/ 10^{-4}{ }^{\circ} \mathrm{C}\end{array}$ & $\begin{array}{l}-\left(\frac{\mathrm{d} T_{\mathrm{g}}}{\mathrm{d} z}\right)_{\mathrm{ob}} \\
/ 10^{-5}{ }^{\circ} \mathrm{C} \mathrm{cm}^{-1}\end{array}$ & $\begin{array}{l}-\Gamma_{\mathrm{g}} \\
/ 10^{-5}{ }^{\circ} \mathrm{C} \mathrm{cm}^{-1}\end{array}$ & $\begin{array}{l}-\left(\frac{\mathrm{d} T_{\mathrm{g}}}{\mathrm{d} z}\right)_{\mathrm{clc}} \\
/ 10^{-5}{ }^{\circ} \mathrm{Ccm}^{-1}\end{array}$ \\
\hline Helium & $\begin{array}{l}2.0 \times 10^{4} \\
530\end{array}$ & $\begin{array}{l}1.0 \pm 0.1 \\
0.94 \pm 0.13\end{array}$ & 0.26 & 1.38 & 1.90 \\
\hline Neon & $\begin{array}{l}5.0 \times 10^{3} \\
660 \\
27 \\
13\end{array}$ & $\begin{array}{l}1.9 \pm 0.2 \\
2.0 \pm 0.2 \\
2.1 \pm 0.2 \\
1.1 \pm 0.3\end{array}$ & 0.53 & 7.56 & 9.50 \\
\hline Argon & $\begin{array}{l}2.7 \times 10^{4} \\
330 \\
73\end{array}$ & $\begin{array}{l}1.25 \pm 0.15 \\
1.5 \pm 0.2 \\
1.6 \pm 0.3\end{array}$ & 0.38 & 14.48 & 18.8 \\
\hline Nitrogen & $\begin{array}{l}2.5 \times 10^{4} \\
930\end{array}$ & $\begin{array}{l}1.1 \pm 0.2 \\
1.2 \pm 0.3\end{array}$ & 0.30 & 7.88 & 9.50 \\
\hline
\end{tabular}

Table 2. Observed $[\Delta T]_{\text {turn }}$, thermal gradient in the gas, estimated $\Gamma_{\mathrm{g}}$, and thermal gradient in the gas which has maximum entropy as an isolated system in a gravitational field.

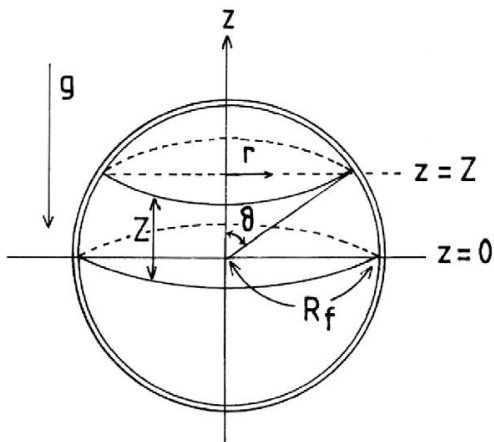

Fig. 5. Coordinate system of a spherical flask.

ence in the outside environment of the flask and $\vartheta$ is the angular coordinate as shown in Figure 5. After a $180^{\circ}$ turn of the flask, the thermal environment of the flask changes to

$$
T_{\text {exf }}=T_{0}+\Delta T_{\text {exf }} \cos (\vartheta+\pi) .
$$

At the time of the $180^{\circ}$ turn of the flask, we consider $t=0$, consequently, the temperature at the wall of the flask $T_{\mathrm{f}}(t, \vartheta)$ can be written as

$$
\begin{aligned}
T_{\mathrm{f}}(t, \vartheta)= & T_{0}-\Delta T_{\operatorname{exf}}\left(1-\frac{2 \lambda_{\mathrm{s}}}{\alpha R_{\mathrm{f}}^{2}}\right) \\
& \cdot \cos \vartheta\left(1-2 \exp \left(-\alpha t / H_{\mathrm{W}}\right)\right),
\end{aligned}
$$

where $\lambda_{\mathrm{s}}, R_{\mathrm{f}}, H_{\mathrm{w}}$ are as shown in Table 1 and

$$
\alpha=4 \sigma \varphi_{\mathrm{f}} T_{0}^{3}+2 \lambda_{\mathrm{s}} / R_{\mathrm{f}}^{2},
$$

where $\varphi_{\mathrm{f}}$ is shown in Table 1 .

The plotted temperature difference $(\Delta T)$ in Figure 4 is given by (10) as

$$
\Delta T=T_{\mathrm{f}}(t, 0)-T_{\mathrm{f}}(t, \pi) .
$$

As shown in Figure 4, the observed temperature difference between the values at $t=0$ and $t>1300 \mathrm{sec}$ is
$5.3 \times 10^{-3}{ }^{\circ} \mathrm{C}$. This temperature difference gives $\Delta T_{\text {exf }}$ in (10) to be $2.8 \times 10^{-3}{ }^{\circ} \mathrm{C}$.

On the other hand, $\Delta T_{\text {exf }}$ is estimated as the temperature at the inside of the drum. The temperature of the outside environment of the drum is given by (1) as $\Delta T_{\text {exD }}=0.25{ }^{\circ} \mathrm{C}$. The temperature at the inside of the drum is given by (2). In this case, the drum is not rotating, and $\alpha_{\mathrm{D}}=1 / 111$. From this estimation $\Delta T_{\text {exf }}$ becomes $2.3 \times 10^{-3}{ }^{\circ} \mathrm{C}$. About a $20 \%$ disagreement between the two estimates of the temperature of the outside environment of the flask might be a good indicator of the accuracy of the estimation in this experiment.

The relaxation time $(\tau)$ for the approach to thermal steady state of the flask given by (10) is

$$
\tau=H_{\mathrm{w}} / \alpha
$$

which corresponds to the observed relaxation time ( $\tau=410 \pm 10 \mathrm{sec}$ ) given by Figure 5. The magnitude of $\alpha$,

$$
\alpha=H_{\mathrm{w}} / 410
$$

is $6.8 \times 10^{-4}$, which is more reliable than the value of $5.6 \times 10^{-4}$ obtained by (11) using the values listed in Table 1. Moreover, in this case, the discrepancy between the two estimations is about $20 \%$.

\subsection{Experimental Results}

All measurements were made under the following setup of the apparatus. The measurement system was placed in the iron cylinder as shown in Figure 2 and evacuated to a pressure lower than $3 \times 10^{-4} \mathrm{~Pa}$. The temperature of the wall of the iron vessel was kept at a constant temperature of $24.0 \pm 0.1{ }^{\circ} \mathrm{C}$. All six heating panels were kept at a constant temperature of 


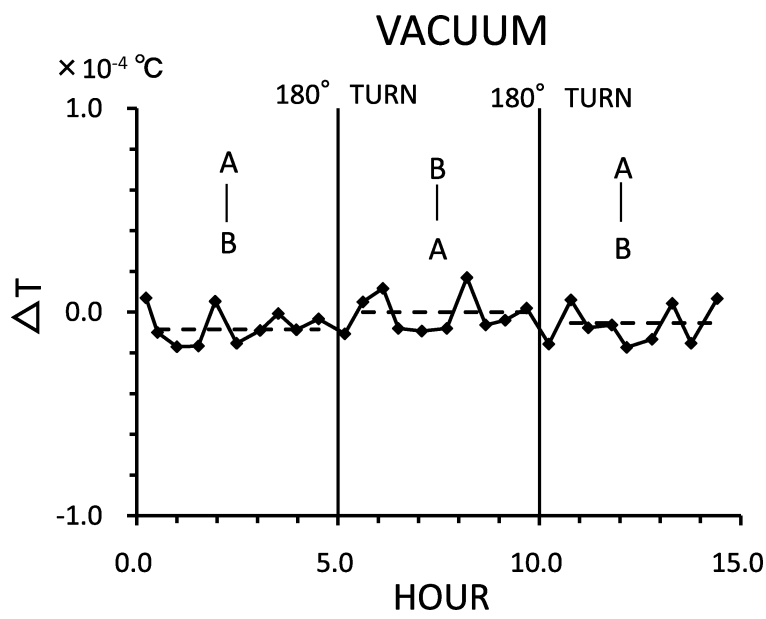

Fig. 6. Observed $\Delta T$ on the flask without the gas. Every $5 \mathrm{~h}$, the flask was turned $180^{\circ}$ and the time of the turn is indicated by the vertical lines. The horizontal dotted lines indicate the mean value of $\Delta T$ measured after the thermal state recovered its steady state after the flask was turned.

$24.20 \pm 0.01{ }^{\circ} \mathrm{C}$. The aluminum drum was rotated continuously with an angular velocity of $0.14 \mathrm{rad} / \mathrm{sec}$. The flask with the gas sample was set with the thermometers A and B placed on a vertical line. After attainment of thermal steady state of the flask, measurements of the temperature difference between the pair of thermistors A and B were made every $180 \mathrm{sec}$. This temperature difference is denoted as $\Delta T$.

$$
\begin{aligned}
\Delta T= & (\text { temperature measured by } \mathrm{A}) \\
& -(\text { temperature measured by } \mathrm{B}) .
\end{aligned}
$$

The mean of ten successive values of $\Delta T$ are plotted in Figures 6, 7, and 8 together with the vertical position of thermistors A and B. The exact temperature difference between the top and the bottom of the flask was set to half the difference of $\Delta T$ values obtained before and after the $180^{\circ}$ turn of the flask. We denoted the value of this observed difference of $\Delta T$ to be $[\Delta T]_{\text {turn }}$. In Figures 6 to 8 , the vertical lines indicate the time of turning of the flask and the horizontal broken lines indicate the mean value of $\Delta T$.

Almost all of the $[\Delta T]_{\text {turn }}$ values listed in Table 2 were obtained by the $180^{\circ}$ turn of the flask with a 5-h interval. In order to confirm that the 5-h interval is sufficient for attaining a thermal steady state, the measurements of $[\Delta T]_{\text {turn }}$ with a turn with 20 -h interval were carried out on the flask with $27 \mathrm{~Pa}$ neon and $2.7 \times 10^{4} \mathrm{~Pa}$ argon.

Figure 6 shows the measured thermal history with a flask without a gas. Figure 7 shows the thermal history

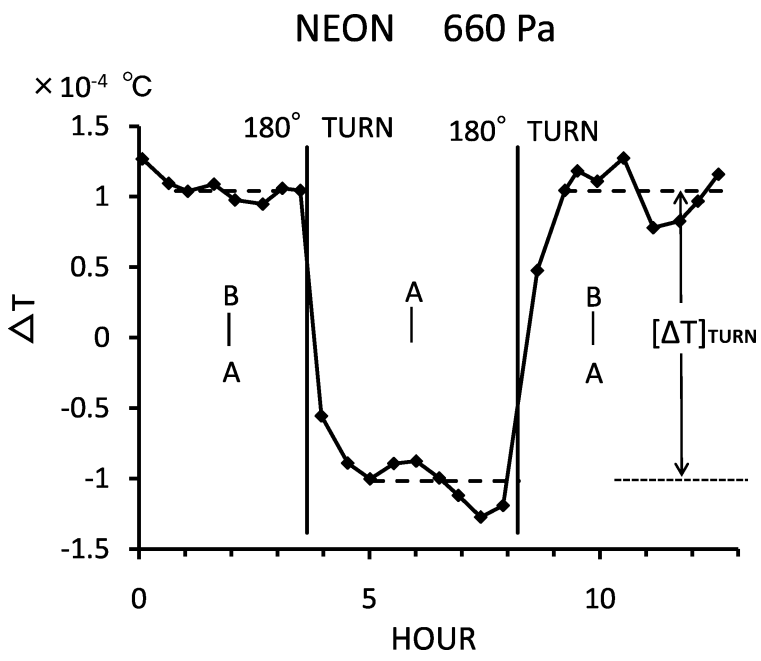

Fig. 7. Observed $\Delta T$ filled with $660 \mathrm{~Pa}$ neon. The process and procedure of the measurements are the same as those used in Figure 6 . The temperature difference $[\Delta T]_{\text {turn }}$ was obtained as the difference in the mean values of $\Delta T$ obtained before and after the $180^{\circ}$ turn of the flask.

measured for the flask with $660 \mathrm{~Pa}$ neon. All $[\Delta T]_{\text {turn }}$ values listed in Table 2 were obtained by observed thermal histories similar to the thermal histories shown in Figures 7 and 8 . Figure 8 shows the thermal history observed for $2.7 \times 10^{4} \mathrm{~Pa}$ argon. In this case, at $10 \mathrm{~h}$ after the start of the measurement, the direction of rotation of the drum was reversed keeping the same angular velocity of rotation. By this reversal of the rotation of the drum, the angular pattern of the non-uniformity of the thermal environment of the flask was shifted by $\pi$, but this did not affect the observed $\Delta T$ values. This is a proof of the thermal uniformity in the environment of the flask during the $\Delta T$ measurements. The observed thermal gradients of the gases in the flask are listed in the fourth column of Table 2 assuming that the temperature of the gas in contact with the wall of the flask is equal to the temperature of the wall of the flask.

As shown in Figures 7 and 8, when the flask contained a gas, the top of the flask was always cooler than the bottom, whereas when the flask contained no gas, no significant temperature difference between the top and the bottom of the flask was observed. Taking the accuracy of the measurements into consideration, the magnitude of the temperature difference between the top and the bottom of the flask depends only on the kind of gas molecules present, and is independent of the pressure of gas within the pressure range from $2.7 \times 10^{4} \mathrm{~Pa}$ to $27 \mathrm{~Pa}$. 
ARGON $2.7 \times 10^{4} \mathrm{~Pa}$

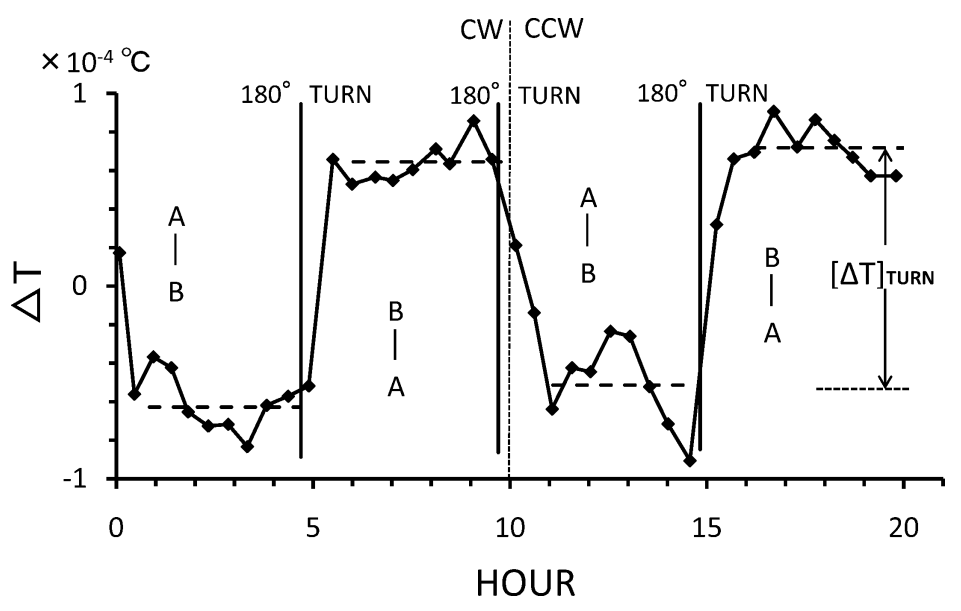

Fig. 8. Observed $\Delta T$ for the flask filled with $2.7 \times 10^{4} \mathrm{~Pa}$ argon. In this case, $10 \mathrm{~h}$ after the start of the measurement, the direction of rotation of the drum was reversed, maintaining a constant angular velocity $\omega=0.14 \mathrm{rad} / \mathrm{sec}$. If the thermal environment of the flask had a temperature difference between the upper and lower sides of the flask, reversal of the direction of rotation of the drum changes the sign of this difference, maintaining the magnitude of the difference. As shown in Figure $8,[\Delta T]_{\text {turn }}$ was not affected by the reversal of the rotation of the drum.

\section{Discussion}

\subsection{Thermal Phenomena Causing the Observed Vertical Temperature Difference at the Flask Wall}

There should be transient thermal disturbances associated with the rotation of the flask, such as heat generation by agitation of gas in the flask and friction at the stem of the flask. These transient thermal disturbances cannot sustain the persistent thermal gradient in the gas. The experimental results gave evidence for the existence of an object with a non-uniform vertical temperature. The object was in a box which was evacuated to a vacuum level better than $3 \times 10^{-4} \mathrm{~Pa}$ and the wall of the box had a homogeneous and steady temperature. The thermal state of this object was also steady and persistent for more then $20 \mathrm{~h}$. The surface of the cold part of this object receives energy from the vacuum environment by thermal radiation, and the surface of the high temperature part of the object emits energy as thermal radiation. These movements of energy at the surface of the object requires persistent energy transportation from the low temperature region to the high temperature region. This peculiar energy flow appeared only when this object contained a gas and only appeared in the vertical direction. This indicates that this peculiar energy flow only appears in the gas induced by the gravitational field. In the gas, energy is transported mainly by molecular heat conduction or by the convective movement of the gas as a mass. Usually, molecular thermal conduction in the gas is consid- ered to be independent of the gravitational field, however, the convective mass motion in the gas requires the presence of gravity.

The amount of transported energy per unit time by this peculiar energy flow in the gas must be equal to the emitting and absorbing energy per unit time at the surface of the vessel which has a temperature difference of $[\Delta T]_{\text {turn }} / 2$. This means that this peculiar flow of energy must be specific to the species of molecules present in the gas and does not depend on the pressure of the gas. This characteristic of the peculiar thermal energy flow is in accord with molecular thermal conduction of the gas but is not in accord with energy transport by convection of the gas. It is known that the velocity of the convective flow of the gas is proportional to the pressure of the gas, and the amount of energy transported by convection in unit time is proportional to the square of the pressure of the gas [9].

In order to clarify the thermal restriction for the appearance of a temperature difference between the top and the bottom of the vessel, we measured $[\Delta T]_{\text {turn }}$ with different settings of the heating panel temperature for the runs with $1 \times 10^{4} \mathrm{~Pa}$ argon and $27 \mathrm{~Pa}$ nitrogen. The observed $[\Delta T]_{\text {turn }}$ values were not appreciably affected when either of the setting temperatures of $\mathrm{H}_{5}$ and $\mathrm{H}_{6}$ deviated by less than $0.01{ }^{\circ} \mathrm{C}$ from $24.20^{\circ} \mathrm{C}$. When the deviation of either of $\mathrm{H}_{5}$ or $\mathrm{H}_{6}$ was $0.02{ }^{\circ} \mathrm{C}$ from $24.20{ }^{\circ} \mathrm{C}$, some of the observed $[\Delta T]_{\text {turn }}$ values decreased by more than half of the normal value. When either $\mathrm{H}_{5}$ and $\mathrm{H}_{6}$ deviated more than $0.04{ }^{\circ} \mathrm{C}$, we could not observe any clear temperature change upon turning the flask. Usually, the expected thermal state due to 
molecular thermal conduction of a gas is not observed by the disturbance of convective flow in the gaseous system. The disappearance of a temperature difference between the top and the bottom of the gas vessel by the slight change in the thermal environment suggests that there is an unknown mode of molecular energy transportation.

At present, we concluded that the observed difference in temperature between the top and the bottom of the flask is created by molecular energy transport of the gas in the flask, because the temperature difference appears only when the flask contains a gas without mass motion and the magnitudes of the observed temperature differences are specific to the molecular species of the gas but are independent of the pressure of the gas in the flask. A gas molecule in a gravitational field changes its vertical velocity due to the gravitational force during flight after collision with another molecule. Then, in the gas without a thermal gradient in a gravitational field, molecules passing downward through a horizontal plane have a higher mean kinetic energy than molecules passing upward through the same horizontal plane. In a gas devoid of mass motion, the number of molecules passing downward through an unit area of the horizontal plane per unit time is equal to that passing upward. We then supposed that there is a downward energy flux $-f_{\mathrm{g}}$ accompanying the gravitational field in the gas. We assumed that in a gravitational field, the vertical energy flux $-F$ in the gas accompanies the energy flux $-f_{\mathrm{g}}$ in addition to the energy flux of the ordinary thermal conduction as

$$
F=-\lambda_{\mathrm{g}} \frac{\mathrm{d} T_{\mathrm{g}}}{\mathrm{d} z}+f_{\mathrm{g}} .
$$

Here, $\lambda_{\mathrm{g}}$ is the ordinary thermal conductivity of the gas, $T_{\mathrm{g}}$ is the temperature of the gas, and $z$ is the vertical coordinate with $z$ increasing upward.

In the equilibrium state of the isolated gaseous system, there must be no thermal energy flow; consequently, the energy flow $F$ becomes zero. Denoting the vertical gradient in the equilibrium state by $\left(\mathrm{d} T_{\mathrm{g}} / \mathrm{d} z\right)_{\text {eq }}$, $F=0$ gives $\left(\mathrm{d} T_{\mathrm{g}} / \mathrm{d} z\right)_{\text {eq }}=f_{\mathrm{g}} / \lambda_{\mathrm{g}}$. At present, we know nothing about the thermal state of the gas in the equilibrium state in a gravitational field experimentally. We can use the value of $\left(\mathrm{d} T_{\mathrm{g}} / \mathrm{d} z\right)_{\text {eq }}$ as an unknown constant $\Gamma_{\mathrm{g}}$. Using this constant $\Gamma_{\mathrm{g}}$, we assumed that the vertical energy flux $F$ in the gas under the gravitational field is given by

$$
F=-\lambda_{\mathrm{g}}\left(\frac{\mathrm{d} T_{\mathrm{g}}}{\mathrm{d} z}-\Gamma_{\mathrm{g}}\right) .
$$

Here, $\lambda_{\mathrm{g}}$ is the ordinary thermal conductivity of the gas, $\left(\mathrm{d} T_{\mathrm{g}} / \mathrm{d} z\right)$ is the vertical thermal gradient in the gaseous system, $z$ is the vertical coordinate increasing upward, and $\Gamma_{\mathrm{g}}$ is a constant which is expected to be the same as the vertical thermal gradient in the equilibrium state of this gas under a gravitational field.

Equation (16) makes it possible to estimate the magnitude of energy flux in the gas even if thermal energy was transported from a cold location to a hot location. Using (16), we can find the relation between the observed values of $[\Delta T]_{\text {turn }}$ and the thermal gradient in the gaseous system which is in an entropy maximum state under the gravitational field.

\subsection{Estimation of $\Gamma_{\mathrm{g}}$ from Observed $[\Delta T]_{\mathrm{turn}}$}

The following calculation gives the magnitude of $\Gamma_{\mathrm{g}}$ from the observed values of $[\Delta T]_{\text {turn }}$ as the requirement for energy conservation. We consider the origin of the coordinate to be the center of the flask and the $z$-axis to be vertical upward with increasing $z$ as shown in Figure 5. When thermal flow per unit area across an horizontal plane of $z=Z$ in the gas is given by (16), the total thermal flux $F_{\mathrm{g}}$ in the flask toward the increasing $z$ direction is

$$
F_{\mathrm{g}}=-\int_{0}^{\sqrt{R_{\mathrm{f}}^{2}-Z^{2}}} \lambda_{\mathrm{g}}\left(\frac{\mathrm{d} T_{\mathrm{g}}}{\mathrm{d} z}-\Gamma_{\mathrm{g}}\right) 2 \pi r \mathrm{~d} r
$$

where $R_{\mathrm{f}}$ is the radius of the flask, $\lambda_{\mathrm{g}}$ is the thermal conductivity of the gas, $T_{\mathrm{g}}$ is the temperature of the gas in the flask, and $r$ is the distance from the center of the flask in the plane $z=Z$ (see Fig. 5).

Along the wall of the flask, across the plane $z=Z$, the thermal energy flux toward increasing $z$ is given by

$$
F_{\mathrm{W}}=\frac{\lambda_{\mathrm{s}}}{R_{\mathrm{f}}} \frac{\mathrm{d} T_{\mathrm{w}}}{\mathrm{d} \vartheta} 2 \pi \sqrt{R_{\mathrm{f}}^{2}-Z^{2}},
$$

where $\lambda_{\mathrm{s}}$ is the same as that used in (10) and $T_{\mathrm{w}}$ is the temperature of the wall of the flask under thermal steady state.

At thermal steady state, the energy flux at the surface of the flask above the plane $z=Z$ toward the outside of the flask is

$$
F_{\mathrm{S}}=\int_{\mathrm{Z}}^{R_{\mathrm{f}}} \sigma \varphi_{\mathrm{f}}\left(T_{\mathrm{w}}^{4}-T_{0}^{4}\right) 2 \pi R_{\mathrm{f}} \mathrm{d} z
$$

where $\varphi_{\mathrm{f}}$ and $\sigma$ are the same as in (11) and (5), and $T_{0}$ is the temperature of the environment of the flask. 
For the conservation of energy, $F_{\mathrm{S}}$ must equal $F_{\mathrm{W}}+$ $F_{\mathrm{g}}$, then:

$$
F_{\mathrm{s}}=F_{\mathrm{g}}+F_{\mathrm{w}}
$$

Computer simulations of the variation of the temperature of the gas in the flask were performed using the assumption that the thermal flux in the gas is given by (16). All the simulations showed that the temperature in the gas approaches a linear function of $z$ and is independent of $r$, in accord with the approach to steady state. Then, at thermal steady state, we can assume

$$
T_{\mathrm{g}}=T_{0}+\left(\frac{\mathrm{d} T_{\mathrm{g}}}{\mathrm{d} z}\right)_{\mathrm{ob}} z .
$$

Neglecting the small difference between the temperature at the wall of the flask and that of the gas in contact with this wall, we can assume $T_{\mathrm{w}}$ as:

$$
T_{\mathrm{w}}=T_{0}+\left(\frac{\mathrm{d} T_{\mathrm{g}}}{\mathrm{d} z}\right)_{\mathrm{ob}} z .
$$

Using (21) and (22), (20) gives the relation between $\Gamma_{\mathrm{g}}$ and the observed thermal gradient in the gas as

$$
\Gamma_{\mathrm{g}}=\left(\frac{\mathrm{d} T_{\mathrm{g}}}{\mathrm{d} z}\right)_{\mathrm{ob}}\left(1+2 \lambda_{\mathrm{s}} /\left(R_{\mathrm{f}} \lambda_{\mathrm{g}}\right)+4 \sigma \varphi_{\mathrm{f}} R_{\mathrm{f}} T_{0}^{3} / \lambda_{\mathrm{g}}\right) .
$$

Using (11) and (13), (23) becomes

$$
\Gamma_{\mathrm{g}}=\left(\frac{\mathrm{d} T_{\mathrm{g}}}{\mathrm{d} z}\right)_{\mathrm{ob}}\left(1+R_{\mathrm{f}} H_{\mathrm{w}} / \tau \lambda_{\mathrm{g}}\right) .
$$

Using the observed vertical thermal gradient in the gas listed in the fourth column of Table 2, (24) gives the values of $\Gamma_{\mathrm{g}}$ in (16) and these values are listed in the fifth column of Table 2 .

\subsection{Comparison of $\Gamma_{\mathrm{g}}$ with the Thermal Gradient in the Gas which is the Entropy Maximum State in a Gravitational Field}

When the gas in the container has a constant total energy including the potential energy, the entropy maximum state of this gaseous system has no thermal gradient [4]. However, when the center of gravity of the gas in the container is fixed in the coordinate of gravity as an isolated gaseous system, the entropy maximum state of this system has a vertical thermal gradient as shown by following calculation. Comparison of this vertical temperature gradient with the value of $\Gamma_{\mathrm{g}}$ calculated using (24) may give the relation between the observed temperature difference between the top and the bottom of the gas vessel and the gravitational field.

Let us consider the state of an ideal gas which is in a column of cross section $S$ and vertical height $L$. We assume $L$ is small, and we can approximate the vertical changes in the molecular density and temperature in the column as a linear functions of $z$ as

$$
\begin{aligned}
& n=n_{0}(1+k z), \\
& T=T_{0}+b z,
\end{aligned}
$$

where $n$ is the molecular density ( $\mathrm{mol} / \mathrm{cc}), T$ is the temperature of the gas in the column, $k$ and $b$ are parameters indicating a vertical change in molecular density and temperature, respectively, $z$ is the vertical coordinate increasing upward, and the $z$ coordinates of the top and the bottom of the column are $L / 2$ and $-L / 2$, respectively. We denote this gaseous system as system A. The gravitational field increases the pressure in accord with the decrease in $z$ in the column as

$$
\mathrm{d} p=-m n g \mathrm{~d} z,
$$

where $p$ is the pressure of the gas in the column, $m$ is the molecular weight of the gas molecule, and $g$ is the acceleration of gravity. If we assume that the gas in the column is an ideal gas, then $\partial p / \partial n=R T, \partial p / \partial T=$ $n R$ and

$$
\frac{\mathrm{d} p}{\mathrm{~d} z}=\frac{\partial p}{\partial n} \frac{\mathrm{d} n}{\mathrm{~d} z}+\frac{\partial p}{\partial T} \frac{\mathrm{d} T}{\mathrm{~d} z} .
$$

These relations give

$$
\frac{\mathrm{d} p}{\mathrm{~d} z}=R T n_{0} k+n R b,
$$

where $R$ is the gas constant. From (27) and (29), we get the following relation between $b$ and $k$ at $z=0$ which is the necessary condition for the gaseous system $\mathrm{A}$ in a gravitational field:

$$
-b=m g / R+T_{0} k
$$

We assume that the vertical coordinate of the center of gravity of system A is fixed in the coordinate system of a gravitational field. Then, the variation of the molecular density in the column does not affect the internal energy of the gas in the system. 
We assume that at first, system A contains $n_{0} S L$ mol ideal gas with a uniform molecular density $n_{0}$ and a uniform temperature $T_{\mathrm{I}}$ in a gravitational field. Realizing this state of the gas requires a large number of partitions in the column. When these partitions are removed, the state of the gaseous system goes to the state indicated by $k, T_{0}$, and $b$, namely, to the state with $n=n_{0}(1+k z)$ and $T=T_{0}+b z$. After this change, $k$, $T_{0}$, and $b$ must satisfy (30). If removal of the partitions in the column was a change in the isolated system, that is this change yields no change in the outside of system A, then the internal energy of the gas in the column maintains a constant value, and the following equation must be satisfied:

$$
\begin{aligned}
& \int_{-L / 2}^{L / 2} C_{\mathrm{v}} m n_{0}\left((1+k z)\left(T_{0}+b z\right)-T_{\mathrm{I}}\right) S \mathrm{~d} z= \\
& C_{\mathrm{v}} m n_{0} S L\left(T_{0}+\frac{L^{2}}{12} k b-T_{\mathrm{I}}\right)=0,
\end{aligned}
$$

where $C_{\mathrm{v}}$ is the heat capacity at constant volume. Equation (31) gives the mean temperature $T_{0}$ as

$$
T_{0}=T_{\mathrm{I}}-\frac{L^{2}}{12} k b .
$$

We can substitute $T_{\mathrm{I}}$ for $T_{0}$ in (30) because the difference between $T_{\mathrm{I}}$ and $T_{0}$ is on the order of $0\left(L^{2}\right)$ as shown by (32). Then, when the value of $k$ is given for an isolated system A, its thermal state $T=T_{0}+b z$ is determined by (30) and (32).

In order to find the entropy maximum state, we can calculate the entropy change from the initial state with $n=n_{0}$ and $T=T_{\mathrm{I}}$ to the final state with $n=n_{0}(1+k z)$ and $T=T_{0}+b z$. This change in the entropy is calculated here in a three step transition. The first step transition is from the initial state to the state with $n=n_{0}(1+k z)$ and $T=T_{\mathrm{I}}$, the second transition is from the last state of the first transition to the state with $n=n_{0}(1+k z)$ and $T=T_{\mathrm{I}}+b z$, and the third transition is from the last state of the second step to the final state.

The first step transition is an isothermal change, then the entropy change $\left(\Delta S_{1}\right)$ by this transition is

$$
\Delta S_{1}=-\frac{W}{T_{\mathrm{I}}}=-\frac{S L^{3}}{24} n_{0} R k^{2}+0\left(L^{5}\right),
$$

where $W$ is the work required to form the final molecular distribution $n=n_{0}(1+k z)$ from the initial uniform distribution by the isothermal transition. The entropy change at the second step transition is

$$
\begin{aligned}
\Delta S_{2}= & \int_{-L / 2}^{L / 2} \int_{T_{\mathrm{I}}}^{T_{\mathrm{I}}+b z} \frac{1}{T} C_{\mathrm{v}} m n_{0}(1+k z) S \mathrm{~d} T \mathrm{~d} z \\
& =\frac{S L^{3}}{12 T_{\mathrm{I}}} C_{\mathrm{v}} m n_{0} b\left(k-\frac{b}{2 T_{\mathrm{I}}}\right)+0\left(L^{5}\right) .
\end{aligned}
$$

The entropy change at the third step transition is calculated as

$$
\begin{aligned}
\Delta S_{3}= & \int_{-L / 2}^{L / 2} \int_{T_{\mathrm{I}}+b z}^{T_{0}+b z} \frac{1}{T} C_{\mathrm{v}} m n_{0}(1+k z) S \mathrm{~d} T \mathrm{~d} z \\
& =-\frac{S L^{3}}{12 T_{\mathrm{I}}} C_{\mathrm{v}} m n_{0} b k+0\left(L^{5}\right) .
\end{aligned}
$$

The entropy change $(\Delta S)$ from the initial state of the system A with $n=n_{0}$ and $T=T_{\mathrm{I}}$ to the final state with $n=n_{0}(1+k z)$ and $T=T_{0}+b z$ is

$$
\begin{aligned}
\Delta S & =\Delta S_{1}+\Delta S_{2}+\Delta S_{3} \\
& =-\frac{S L^{3}}{24} n_{0}\left(R k^{2}+C_{\mathrm{v}} m \frac{b^{2}}{T_{\mathrm{I}}^{2}}\right)+0\left(L^{5}\right) .
\end{aligned}
$$

The entropy maximum state of the gaseous system A has the value $k$ determined by $(\mathrm{d} \Delta S / \mathrm{d} k)=0$ as

$$
k=-\frac{C_{\mathrm{v}} m^{2} g}{T_{\mathrm{I}} R\left(R+C_{\mathrm{v}} m\right)},
$$

where (30) was used for the differentiation of (36). With this $k$, (30) gives $b$ as

$$
\left(\frac{\mathrm{d} T_{\mathrm{g}}}{\mathrm{d} z}\right)_{\mathrm{clc}}=-\frac{g}{C_{\mathrm{v}}+R / m},
$$

where the left side of (38) is $b$ which is the thermal gradient in the gas at the entropy maximum state. This thermal gradient is identical to that in the convective equilibrium state [5]. For a comparison of these values of the thermal gradient with the observed $\Gamma_{\mathrm{g}}$, the values of the thermal gradient given by (38) are listed in the last column of Table 2.

Taking the numerical accuracy of the observed values of $[\Delta T]_{\text {turn }}$ and that of the estimation by (24) into consideration, we conclude that the values of $\Gamma_{\mathrm{g}}$ shown in the fifth column of Table 2 agree with the thermal gradient of the gas in the equilibrium state in a gravitational field shown in the last column of Table 2. This coincidence of $\Gamma_{\mathrm{g}}$ with the thermal gradient given 
by (38) makes the assumption given by (16) plausible but the assumption is still unusual. We suggest further verification of the experimental results of this study and further investigations into the assumption made by (16).

\section{Acknowledgement}

The measurements in this work were carried out over 10 years after 1988 in the Radiocarbon Dating Laboratory, Faculty of Science, Gakushuin University. The author thanks all those who arranged

[1] H. Craig, Y. Horibe, and T. Sowers, Science 242, 4885 (1988).

[2] T. Sowers, M. Bender, and D. Raynaud, J. Geophys. Res. 94, 5137 (1989).

[3] H. Craig and R. C. Wiens, Science 271, 1706 (1996).

[4] C. Y. Adachi, K. Kawamura, L. Armi, and R. F. Keeling, Science 311, 1429 (2006).

[5] S. Ishidoya, S. Sugawara, S. Morimoto, S. Aoki, and T. Nakazawa, Geophys. Res. Lett. 35, 3811 (2008). colloquiums for discussions of the experimental results in this study in the Departments of Physics and Chemistry, Gakushuin University, in the Department of Astrophysics, A.F. Ioffe Physical-Technical Institute, in the Radiocarbon Dating Laboratory, University of Helsinki, in the Department of Astronomy, Stanford University, in the Department of Space Physics, California Institute of Technology, in the Department of Physics, University of Kansas, and in the Space Physics Center, University of Arizona.

[6] W. Schroer, Z. Naturforsch. 46a, 187 (1991).

[7] A. Sommerfeld, Thermodynamics and Statistical Mechanics, Academic Press Inc., New York 1955, p. 360.

[8] E. Fermi, Thermodynamics, Dover Pub. Inc., Dover 1956, p. 28.

[9] W. H. Furry, R. C. Jones, and L. Onsager, Phys. Rev. 55, 1083 (1939). 